\title{
Cross-sectional Diameter at End Ventricular Diastole
}

National Cancer Institute

\section{Source}

National Cancer Institute. Cross-sectional Diameter at End Ventricular Diastole. NCI

Thesaurus. Code 127545.

The cross sectional diameter measured at end ventricular diastole. 